\title{
Disappointing reliability of pulsatility indices to identify candidates for magnetic resonance imaging screening in population-based studies assessing prevalence of cerebral small vessel disease
}

\author{
Oscar H. Del Brutto, Robertino M. Meraㄹ, María de la Luz Andrade², Pablo R. Castillo³, Mauricio Zambrano ${ }^{4}$, \\ Juan A. Nader ${ }^{1}$ \\ School of Medicine, Universidad Espiritu Santo - Ecuador, ${ }^{1}$ Department of Gastroenterology, Vanderbilt University, Nashville, TN, \\ ${ }^{2}$ Department of Neurology, Hospital Medica Sur, Mexico City, Mexico, ${ }^{3}$ Mayo Clinic College of Medicine, Sleep Disorder Center, Jacksonville, Fl, \\ ${ }^{4}$ Community Center, the Atahualpa Project, Atahualpa, Ecuador
}

\begin{abstract}
Background: Diagnosis of cerebral small vessel disease (SVD) is a challenge in remote areas where magnetic resonance imaging (MRI) is not available. Hospital-based studies in high-risk or stroke patients have found an association between the pulsatility index (PI) of intracranial arteries - as derived from transcranial Doppler (TCD) - and white matter hyperintensities (WMH) of presumed vascular origin. We aimed to assess the reliability of cerebral pulsatility indices to identify candidates for MRI screening in population-based studies assessing prevalence of SVD. Methods: A representative sample of stroke-free Atahualpa residents aged $\geq 65$ years investigated with MRI underwent TCD. Using generalized linear models, we evaluated whether the PI of major intracranial arteries correlate with WMH (used as a proxy of diffuse SVD), after adjusting for demographics and cardiovascular risk factors. Results: Out of 70 participants (mean age $70.6 \pm 4.6$ years, $57 \%$ women), 28 (40\%) had moderate-to-severe WMH. In multivariate models, there were no differences across categories of WMH in the mean PI of middle cerebral arteries ( $1.10 \pm 0.16$ vs. $1.22 \pm 0.24, \beta$ : $0.065,95 \%$ confidence interval $(C I):-0.084-0.177, P=0.474)$ or vertebrobasilar arteries $(1.11 \pm 0.16$ vs. $1.29 \pm 0.27, \beta$ : $0.066,95 \% \mathrm{CI}:-0.0024-0.156, P=0.146)$. Conclusions: Cerebral PI should not be used to identify candidates for MRI screening in population-based studies assessing the burden of SVD.
\end{abstract}

Key words: Population-based studies, pulsatility index, small vessel disease, transcranial Doppler, white matter hyperintensities

\section{Introduction}

Stroke prevalence is increasing in Latin America. ${ }^{[1]}$ Identification of pathogenetic mechanisms underlying stroke is important for the implementation of intervention strategies in apparently healthy individuals before catastrophic cerebrovascular events occur. A recent population-based study conducted in older adults living in rural Ecuador showed that two-thirds of these persons

\begin{tabular}{|l|l|}
\hline \multicolumn{2}{|c|}{ Access this article online } \\
\hline Quick Response Code: & Website: \\
\hline & www.ruralneuropractice.com \\
\cline { 2 - 3 } & \\
\hline
\end{tabular}

have magnetic resonance imaging (MRI) evidence of silent cerebral small vessel disease (SVD). ${ }^{[2]}$ However, more studies are needed to confirm the burden of SVD in underserved Latin American populations and its role in stroke incidence.

Diagnosis of SVD requires the use of brain MRI, which is not available in rural areas. Therefore, efforts should be directed to find portable and reliable screening diagnostic tools that may help identify candidates for MRI screening. In this view, transcranial Doppler (TCD) examination has been proposed as an inexpensive alternative. Calculation of the pulsatility index (PI) of the middle cerebral artery (MCA) has been correlated with white matter hyperintensities (WMH) of presumed vascular origin and may disclose the presence of silent SVD. ${ }^{[3-5]}$ Since poor acoustic insonation through

Address for correspondence:

Dr. Oscar H. Del Brutto, School of Medicine, Universidad Espíritu Santo, Ecuador. E-mail: oscardelbrutto@hotmail.com 
transtemporal windows is a limitation of TCD - and may preclude its use for mass screening - it has been suggested that PI calculations of vertebrobasilar (VB) arteries also correlate with $\mathrm{WMH}$, based on the concept that SVD is a diffuse process affecting all intracranial arteries. ${ }^{[4]}$ Here, we aimed to assess the reliability of PI to identify candidates for MRI testing in community-dwelling older adults living in Atahualpa, a remote rural village where previous epidemiological studies using MRI have been conducted. $[2,6]$

\section{Methods}

The Institutional Review Board of Hospital-Clínica Kennedy, Guayaquil (FWA 00006867) approved the protocol and the written informed consent. Atahualpa was selected as a village representative of rural coastal Ecuador. More than $95 \%$ of the population belong to the Native/Mestizo ethnic group (Amerindians) and their living characteristics are homogeneous, as detailed elsewhere. ${ }^{[2,6]}$

Eighty-two randomly selected individuals were invited to the Community Center of the Atahualpa Project for the practice of TCD. They were selected by the use of the random integer generator (https://www.random. org/integers/). These 82 individuals represented the $50 \%$ of 164 Atahualpa residents fulfilling the following criteria: (1) Age $\geq 65$ years, (2) clinical stroke-free status, (3) brain MRI performed in the previous 6 months, (4) patency of all major intracranial vessels on magnetic resonance angiography, and (5) no atrial fibrillation. Neuroimaging studies had been performed with a Philips Intera 1.5T MRI machine (Philips Medical Systems, the Netherlands) at Hospital-Clínica Kennedy, using a protocol described elsewhere. ${ }^{[2,6]}$ For this study, primary interest focused on the presence of $\mathrm{WMH}$, defined as lesions appearing hyperintense on T2-weighted images that remained bright on Fluid attenuated inversion recovery (without cavitation) and graded in mild, moderate and severe according to the modified Fazekas scale ${ }^{[7]}$ According to this scale, mild cases include periventricular caps or thin lesions and punctate hyperintensities in subcortical white matter. In moderate cases, there is a smooth periventricular halo and subcortical foci begin to merge. Severe cases characterize by extension of periventricular lesions into the subcortical white matter and large confluent subcortical foci.

As previously detailed, ${ }^{[8]}$ the cardiovascular health (CVH) status of individuals enrolled in the Atahualpa Project had been evaluated by the use of the seven metrics proposed by the American Heart Association, including smoking status, body mass index, physical activity, diet, blood pressure, fasting glucose, and total cholesterol blood levels.

With the use of a SONARA portable TCD system (VIASYS Healthcare, Inc. Madison, WI, USA) and a 2-MHz probe, two experienced sonographers performed TCDs following a power motion mode Doppler/spectral TCD insonation protocol..$^{[9]}$ Information provided by insonation through transtemporal and transforaminal windows were analyzed. We recorded peak systolic velocity (PSV), end-diastolic velocity (EDV), mean flow velocity (MFV) and the PI of both MCAs and VB arteries. The PI of each of these vessels was calculated according to the Gosling equation (PSV-EDV/MFV). According to Kidwell et al., ${ }^{[5]}$ a mean MCA PI was calculated by averaging both MCA PI (unless the person only had one adequate transtemporal window, in which case unilateral MCA PI was measured), and a mean VB PI was calculated by averaging the PI of the basilar and vertebral arteries. We constructed generalized linear models (with a Gaussian link) to evaluate the association between the PI of the investigated arteries and WMH severity, after adjusting for age, sex, and $\mathrm{CVH}$ status. Statistical analyses were carried out by using STATA version 13 (College Station, TX, USA).

Table 1: Correlation between the MCA PI and the mean VB PI across categories of WMH severity, in univariate and multivariate models. The multivariate model was adjusted for age, sex, and cardiovascular health status. Loss of significance in multivariate models was mainly related to the strong effect of age

\begin{tabular}{|c|c|c|c|c|c|}
\hline & $\begin{array}{l}\text { Total } \\
\text { series }\end{array}$ & $\begin{array}{l}\text { None-to- } \\
\text { mild WMH }\end{array}$ & $\begin{array}{l}\text { Moderate-to- } \\
\text { severe WMH }\end{array}$ & $\begin{array}{l}\text { Univariate analysis } \\
\text { of variance }\end{array}$ & $\begin{array}{c}\text { Generalized linear } \\
\text { model }\end{array}$ \\
\hline $\begin{array}{l}\text { Mean MCA PI } \\
(n=44)\end{array}$ & $1.15 \pm 0.21$ & $1.10 \pm 0.16$ & $1.22 \pm 0.24$ & $P=0.053$ & $\begin{array}{c}\beta: 0.065 \\
95 \% \text { Cl: }-0.084-0.177 \\
P=0.474\end{array}$ \\
\hline $\begin{array}{l}\text { Mean VB PI } \\
(n=70)\end{array}$ & $1.18 \pm 0.23$ & $1.11 \pm 0.16$ & $1.29 \pm 0.27$ & $P=0.001$ & $\begin{array}{c}\beta: 0.066 \\
95 \% \mathrm{Cl}:-0.024-0.156 \\
P=0.146\end{array}$ \\
\hline
\end{tabular}

MCA PI: Middle cerebral artery pulsatility index, VB: Vertebrobasilar, WMH: White matter hyperintensities, Cl: Confidence interval 


\section{Results}

Out of 82 invited individuals, 73 (89\%) agreed to participate. Three of them were excluded due to insonation problems through all acoustic windows. In $26(37 \%)$ of the remaining persons (20 of whom were women), poor insonation through both transtemporal windows precluded MCA PI calculations, but remained in the study because VB PI could be calculated. Mean age of the 70 participants was $70.6 \pm 4.6$ years, 40 (57\%) were women and $60(86 \%)$ had a poor CVH status. Individual cardiovascular risk factors more often in the poor range included a blood pressure $\geq 140 / 90 \mathrm{mmHg}$ in 47 individuals (67\%), total cholesterol blood levels $\geq 240 \mathrm{mg} / \mathrm{dL}$ in nine (13\%) and fasting glucose levels $\geq 126 \mathrm{mg} / \mathrm{dL}$ in six (9\%).

Mean PI in the right $(1.14 \pm 0.22)$ versus the left MCA $(1.13 \pm 0.19)$ were not different $(P=0.774)$. Similarly, there no significant differences in the mean PI of the right vertebral $(1.19 \pm 0.29)$, the left vertebral $(1.2 \pm 0.28)$ and the basilar arteries $(1.15 \pm 0.26), P=0.529$.

Moderate-to-severe WMH were noticed in 28 (40\%) cases. These persons were older (mean age $77.4 \pm 7$ vs. $72.2 \pm 5.5$ years, $P=0.003$ ) and had more often a poor CVH status $(96 \%$ vs. $79 \%, P=0.043)$ than those with none-to-mild $\mathrm{WMH}$, but there were no sex-related differences across groups. Univariate analysis showed that mean MCA PI and VB PI were higher in persons with moderate-to-severe than in those with none-to-mild WMH. However, such differences were lost in multivariate models [Table 1].

\section{Discussion}

In this representative sample of older adults living in a rural Latin American population, the PI of major intracranial arteries do not correlate with WMH severity after adjusting for confounding variables. A high PI may not only reflect distal cerebrovascular resistance (and thus, SVD) but may also occur as the result of large artery stiffness or other hemodynamic factors. ${ }^{[10]}$ Because of its complex nature, PI is not useful to assess SVD prevalence and should not be used alone as a proxy for SVD. A potential limitation of the present study is the sample size, which may not be representative of other Latin American villages. Further cooperative studies are needed to settle the role of TCD in mass screening of MRI candidates for SVD assessment.

\section{References}

1. Lavados PM, Hennis AJ, Fernandes JG, Medina MT, Legetic B, Hoppe A, et al. Stroke epidemiology, prevention, and management strategies at a regional level: Latin America and the Caribbean. Lancet Neurol 2007;6:362-72.

2. Del Brutto OH, Mera RM, Del Brutto VJ, Zambrano M, Lama J. White matter hyperintensities of presumed vascular origin: A population-based study in rural Ecuador (The Atahualpa Project). Int J Stroke 2015;10:372-5.

3. Heliopoulos I, Artemis D, Vadikolias K, Tripsianis G, Piperidou C, Tsivgoulis G. Association of ultrasonographic parameters with subclinical white-matter hyperintensities in hypertensive patients. Cardiovasc Psychiatry Neurol 2012;2012:616572.

4. Xiong YY, Mok V, Wong A, Leung T, Chen XY, Chu WC, et al. Evaluation of age-related white matter changes using transcranial Doppler ultrasonography. J Neuroimaging 2013;23:53-7.

5. Kidwell CS, el-Saden S, Livshits Z, Martin NA, Glenn TC, Saver JL. Transcranial Doppler pulsatility indices as a measure of diffuse small-vessel disease. J Neuroimaging 2001;11:229-35.

6. Del Brutto OH, Mera RM, Zambrano M, Lama J. Incompleteness of the Circle of Willis correlates poorly with imaging evidence of small vessel disease. A population-based study in rural Ecuador (the Atahualpa project). J Stroke Cerebrovasc Dis 2015;24:73-7.

7. Pantoni L, Basile AM, Pracucci G, Asplund K, Bogousslavsky J, Chabriat $\mathrm{H}$, et al. Impact of age-related cerebral white matter changes on the transition to disability-The LADIS study: Rationale, design and methodology. Neuroepidemiology 2005;24:51-62.

8. Del Brutto OH, Santamaría M, Ochoa E, Peñaherrera E, Santibáñez R, Pow-Chon-Long F, et al. Population-based study of cardiovascular health in Atahualpa, a rural village of coastal Ecuador. Int J Cardiol 2013;168:1618-20.

9. Alexandrov AV, Demchuk AM, Burgin WS. Insonation method and diagnostic flow signatures for transcranial power motion (M-mode) Doppler. J Neuroimaging 2002;12:236-44.

10. Webb AJ, Simoni M, Mazzucco S, Kuker W, Schulz U, Rothwell PM. Increased cerebral arterial pulsatility in patients with leukoaraiosis: Arterial stiffness enhances transmission of aortic pulsatility. Stroke 2012;43:2631-6.

How to cite this article: Brutto $\mathrm{OH}$, Mera RM, Andrade Md, Castillo PR, Zambrano M, Nader JA. Disappointing reliability of pulsatility indices to identify candidates for magnetic resonance imaging screening in population-based studies assessing prevalence of cerebral small vessel disease. J Neurosci Rural Pract 2015;6:336-8.

Source of Support: Nil. Conflict of Interest: None declared. 